\title{
Radiological Imaging Findings of Rhino-Orbito-Cerebral Mucormycosis in COVID and Post-COVID Patients - A Descriptive Study of 200 Patients
}

\author{
Yasmeen Usmani ${ }^{1}$, Bitthal Shukla², Shishir Kumar ${ }^{3}$, Monika Dubey ${ }^{4}$, Mohd. Asif ${ }^{5}$ \\ 1, 2, 3,4,5 Department of Radiology, Lala Lajpat Rai Memorial Medical College, Meerut, Uttar Pradesh, India.
}

\section{ABSTRACT}

\section{BACKGROUND}

Rhino-orbito-cerebral mucormycosis (ROCM) is a life-threatening infection caused by saprophytic fungi. The purpose of this study was to describe common radiographic patterns that may be useful in predicting the diagnosis and assessing the extent of involvement of rhino-orbito-cerebral mucormycosis in Covid and post Covid patients.

\section{METHODS}

The case records of patients with rhino-orbito-cerebral mucormycosis were reviewed. This included 200 patients of which 122 were males and 78 females, with a minimum age of 22 to a maximum of 80 years old. Computed tomography (CT) and / or magnetic resonance imaging (MRI) images were retrieved from the picture archiving and communication system (PACS) and analysed. Statistical analysis was performed using descriptive statistics.

\section{RESULTS}

All the patients showed sinusitis and ophthalmological symptoms. CT and MR imaging showed predominant involvement of the maxillary $(196,98 \%)$ and ethmoid $(194,97 \%)$ sinuses. Involvement of the orbit $(186,93 \%)$ and face $(114,57$ $\%)$ preceded involvement of the pterygopalatine fossa $(90,45 \%)$ and brain $(52,26$ $\%)$. CT showed minimally enhancing hypodense soft tissue thickening as the predominant finding in involved areas, while MRI showed T2 isointense to mildly hypointense soft tissue thickening and heterogeneous post-contrast enhancement as the main finding. In patients with extra sinus extension, bone erosion was seen in 80 patients $(40 \%)$ but few $(20,10 \%)$ of the patients showed extension across grossly intact appearing bones.

\section{CONCLUSIONS}

In the context of Covid-19 with immunosuppression, a pattern of the nasal cavity, maxillary sinus, ethmoid cells, and orbit inflammatory lesions should prompt the diagnosis of mucormycosis. Progressive and rapid involvement of the cavernous sinus, vascular structures and intracranial contents can occur. Multiplanar magnetic resonance imaging and computed tomography show anatomic involvement, helping in surgery planning.

\section{KEY WORDS}

Rhino-Orbito-Cerebral mucormycosis, Imaging Findings, MRI, Neuroradiology

\author{
Corresponding Author: \\ Dr. Bitthal Shukla, \\ Room no. S2, Senior Boys Hostel, \\ L. L. R. M. Medical College, Meerut, \\ Uttar Pradesh, India. \\ E-mail: bitthal.shukla1@gmail.com
}

DOI: $10.14260 / \mathrm{jemds} / 2021 / 703$

How to Cite This Article:

Usmani Y, Shukla B, Kumar S. et al. Radiological imaging findings of rhinoorbito-cerebral mucormycosis in covid and post-covid patients - a descriptive study of 200 patients. J Evolution Med Dent Sci 2021;10(39):3470-3473, DOI: 10.14260/jemds/2021/703

Submission 05-06-2021, Peer Review 08-09-2021, Acceptance 14-09-2021, Published 27-09-2021.

Copyright (c) 2021 Yasmeen Usmani et al. This is an open access article distributed under Creative Commons Attribution License [Attribution 4.0 International (CC BY 4.0)] 


\section{BACKGROUND}

Covid-19 (coronavirus disease 2019) is a highly infectious respiratory disease caused by severe acute respiratory syndrome coronavirus 2 (SARS-CoV-2). The origin of the first case was reported from Wuhan; China, in December 2019 and subsequently there was a rapid global spread resulting in it being declared as a "pandemic" on 11th March 2020. The first case reported in India was on January 29, 2020. The patients usually presented with dry cough, breathlessness, fever and other nonspecific prodromal symptoms progressing up to severe respiratory distress syndrome. Secondary complications with Covid-19 include pulmonary and venous embolism and acute cardiac injury leading to myocardial ischemia and myocarditis. Intracranial complications in the form of stroke were also seen. In the first wave, the incidence of orbital and cerebral complications due to fungal aetiology was not seen. The second wave of Covid-19 was reported to have started from 10 February 2021 in India. In the $2^{\text {nd }}$ wave, rhino-orbito-cerebral mucormycosis was widely prevalent in both active and recovered Covid patients. The infection is caused by a saprophytic opportunistic fungus which usually does not cause any disease. ${ }^{1}$ However; in Covid-19 patients the infection had a high incidence. This was especially seen in diabetic patients. This was due to the greater availability of glucose to the pathogen and lower response of T-cells. Also, widespread and indiscriminate use of immunosuppressants such as corticosteroids decreases immunity to opportunistic pathogens such as fungi (mucormycosis). Clinically, presenting symptoms are non-specific and varied including headache, low-grade fever, facial and scalp swelling and orbital or paranasal sinus syndrome with epistaxis. The radiological and clinical findings included rhino-sinusitis (involving bilateral nasal cavities, maxillary, frontal, ethmoidal and sphenoidal sinuses). Air foci were noted on CT. Erosion of maxillary and sphenoid bones was also noted. After infection of the nasal cavity and paranasal sinuses, the fungi can cause a necrotizing vasculitis that extends rapidly into infratemporal and pterygopalatine fossae, deep face, orbits, cranial cavity and brain through skull base partitions and foramina. ${ }^{2}$ Inflammation and oedema of orbital tissues in both intraconal and extraconal compartments involving extraocular muscles with proptosis were seen. Inflammation of surrounding muscles of mastication (temporalis, masseter and medial and lateral pterygoids) was noted. Vascular involvement in the form of cavernous sinus and internal carotid artery thrombosis was seen. Inflammation and infection of the orbital apex were noted with optic neuritis. Intracranial involvement was noted in the form of cerebritis and cerebral abscesses predominantly in the frontal regions along with infarcts due to vascular changes. The disease has a variable prognosis. When limited involvement of the paranasal sinuses is present, survival rates are between $50 \%$ and $80 \%{ }^{3}$ However, when brain invasion has occurred, mortality is greater than $80 \%$. Because of its aggressive and lethal nature, it must be recognized early and treated aggressively. Imaging through MRI and CT plays an important role in this. We reviewed the neuroimaging findings in a series of 200 patients with rhino-orbito-cerebral mucormycosis to establish common radiographic patterns that may be useful in predicting the early diagnosis and assessing the extent of this infection by MRI/CT.

\section{METHODS}

This is a case series type of study involving patients admitted in nodal centre LLRM Medical College, Meerut, (U. P.), India which includes referred cases from adjoining districts Saharanpur, Khatauli, Bulandshahr and Meerut. 200 Covid and Post Covid patients had been admitted from 22 April, 2021 to 15 June, 2021. All of them underwent imaging by CT/MRI to study the presence and extent of the study. 172 (86\%) cases were known cases of RT-PCR positive for Covid, and rest $28(14 \%)$ cases were RT-PCR negative with typical symptoms of influenza-like illness. $90 \%$ of total cases included in the study were not vaccinated for Covid-19, $8 \%$ were vaccinated with single-dose and only $2 \%$ were completely vaccinated with two doses. 56 (28\%) Covid positive cases were in home isolation and 144 (72\%) Covid positive cases were hospitalised. Out of the hospitalized patients, $160(80 \%)$ cases required oxygen in which mode of oxygen delivery was by simple face mask in 140 (70\%) patients, 36 (18\%) by non re-breather mask (NRBM) and 24 (12\%) by non-invasive ventilation (NIV). 160 (80\%) patients underwent steroid treatment and $40(20 \%)$ with no steroid therapy. 156 (78 \%) had diabetes mellitus, out of which 50 (32\%) were newly detected and 40 (22\%) had no diabetes. $13(6.67 \%)$ patients of both diabetic and nondiabetic types had hematologic conditions and concomitant immunocompromised states. The patients included 122 males and 78 females. The minimum age of patients was 22 years and the maximum was 80 years. The computed tomography (CT) and magnetic resonance imaging (MRI) images were retrieved from the picture archiving and communication system (PACS). CT scan was performed on a GE Optima 64 slice machine using a routine CT Paranasal sinus (PNS) protocol with $130 \mathrm{kVP}$ and $150-220 \mathrm{~mA}$ tube current. Conventional MR Neck imaging included axial, coronal and sagittal T1 weighted (TR/TE 40/12 ms), T2weighted images (TR/TE 4000/100 ms) and fat-suppressed post-contrast $\mathrm{T} 1$ weighted images were acquired. MRI imaging was performed using $1.5 \mathrm{~T}$ Philips Achieva. Contrast MRI with gadolinium was done in 26 patients.

\section{Image Interpretation}

The sinuses showing opacification on CT or MRI were recorded in each case. The appearance on plain T1 and T2 images was documented and involvement of any extra sinus structures including orbit, face, pterygopalatine fossa, masticator space, brain and cavernous sinus was noted as fat stranding and soft tissue extension similar in appearance to the intrasinus soft tissue. Orbital cellulitis was seen as stranding in the retrobulbar fat. On post-contrast, MRI, the type of contrast enhancement was seen. Any complications like arterial thrombosis were assessed. Cavernous sinus involvement was seen as thickening and non-enhancement on post-contrast scans with the presence of abnormal surrounding soft tissue. Patients with intracranial extension 
were evaluated for dural enhancement, presence of extradural collections, infarcts, cerebritis and intracerebral abscess. The presence of bone involvement was evaluated on the CT.

The patients in the study were divided into three groups based on the extent of regional involvement according to the classification suggested by Rupa et al. Table 1.4

\begin{tabular}{|c|c|c|c|}
\hline Stage & Areas Involved & Number & Percentage (\%) \\
\hline Stage 1 & Nose \& paranasal sinuses alone & 100 & 50 \\
\hline Stage 2 & $\begin{array}{l}\text { Paranasal sinuses with immediate } \\
\text { adjacent areas which are surgically } \\
\text { resectable with minimal morbidity e.g. } \\
\text { orbit (extraconal), palate \& oral cavity }\end{array}$ & 60 & 30 \\
\hline Stage 3 & $\begin{array}{l}\text { Intracranial extension } \\
\text { (extradural/intracerebral) or partially } \\
\text { resectable with extension to } \\
\text { pterygopalatine fossa, cavernous sinus, } \\
\text { cheek and periorbital region }\end{array}$ & 40 & 20 \\
\hline Total & & 200 & 100 \\
\hline
\end{tabular}

\section{Analytic Methods}

Mainly descriptive studies were used.

\section{RESULTS}

\section{Clinical observations}

The study had 122 males and 78 females with a minimum age of 22 years and maximum age of 80 years (Mean $=49$ years). $160(80 \%)$ patients underwent steroid treatment and 40 (20 $\%)$ patients with no steroid use. 156 (78 \%) had diabetes mellitus with 44 (22\%) patients having no diabetes. 13 (6.67 $\%$ patients of both diabetic and non-diabetic types had hematologic conditions and concomitant immunocompromised states.

The patients presented with symptoms of headache $(175$, $87.5 \%)$, rhinorrhea $(137,68.5 \%)$, facial oedema (137, $68.5 \%)$, pain in face $(129,64.5 \%)$, fever $(100,50 \%)$, decrease in sight $(41,20.5 \%)$ and bleeding from nose $(17,8.5$ $\%)$.

\section{Radiological Imaging}

Nose and Paranasal Sinus Disease

The most commonly involved sinus was maxillary (196, 98 $\%$ ). Multiple sinus disease was seen in 194 patients (97\%). When multiple sinuses were involved, ethmoid, sphenoid and maxillary sinus were affected in 164 patients (84\%). More patients had involvement of both side sinuses (79\%) than on one side (21\%). The spectrum is shown in Table 2.

106 (53\%) had isodense muscle/brain lesions. 52 (26\%) lesions were hypodense. 40 (20\%) had lesions that were hyperdense which were due to retained secretions.

\section{CT}

Infection was infiltrative in patients who showed fat stranding in facial, pterygopalatine and orbital areas with soft tissue lesion extension. Bony structures were normal in 119 $(59.5 \%)$ patients with few of them having an extension into soft tissues with normal bone. 81 (40.5\%) patients showed bone involvement in the form of erosions, sclerosis and lytic areas.
On CT nasal cavity, involvement was seen in 132 (66\%) cases. This was seen in the form of turbinate hypertrophy with the mild surrounding fluid.

\section{Magnetic Resonance Imaging}

The lesions were isointense or showed mild hypointensity. T2W MRI showed three variations:

Isointense or hypointense in 73 (36.5\%) patients.

Heterogeneous in 67 (33.5\%) patients.

Hyperintense with fluid in 36 (30\%).

In 3 cases with sphenoid sinus involvement, soft tissue extension was seen involving cavernous sinuses which were hypointense on T2WI. 2 similar patients with maxillary sinus involvement had an extension in the pterygopalatine fossa which was also hypointense on $\mathrm{T} 2$.

Diffusion-Weighted MRI was done in 104 patients out of 200. 71 of these 104 patients showed bright signals on DWI (restriction). This was mainly due to intracranial extension or collection in soft tissues as shown in Table 2

\section{Enhancement Pattern}

CE MRI was done in 26 cases. Three types of enhancement were seen:

Homogeneous in 8 cases (30.7\%)

Heterogeneous enhancement in 9 cases (34.6\%)

Central dark with peripheral enhancement in 9 (34.6\%) patients.

Enhancement was visualized on fat suppressed images. A black turbinate sign given by Safder et al. was seen in 4 out of 26 patients. Enhancement of dura was observed in 17 patients (65.38\%). Enhancement of leptomeninges was seen in 9 patients (34.61\%).

\section{Involvement of Surrounding Tissues}

The most commonly involved structure was orbit in $93 \%$ of patients. The face was involved in $56 \%$ of cases followed by pterygopalatine fossae, temporal and infratemporal areas, muscles of mastication, the apex of the orbit, surrounding masticator areas, cavernous sinuses and internal carotid arteries. Further details are given in Table 3.

\begin{tabular}{|c|c|c|c|}
\hline CT Feature & N (\%) & MR Feature & N (\%) \\
\hline \multirow{2}{*}{$\begin{array}{l}\text { Mucosal } \\
\text { thickening }\end{array}$} & \multirow{2}{*}{$200(100 \%)$} & T1 W signal & \\
\hline & & Iso/Hypo intense & $200(100 \%$ \\
\hline \multirow{8}{*}{ Osseous erosion } & \multirow{8}{*}{$80(40 \%)$} & $\mathrm{T} 2 \mathrm{~W}$ signal & \\
\hline & & Isointense/Hypointense & $72(36 \%)$ \\
\hline & & Heterogeneous & $64(32 \%)$ \\
\hline & & Hyperintense & $64(32 \%)$ \\
\hline & & Enhancement pattern in CEMRI $(\mathrm{N}=26)$ & \\
\hline & & Intense homogenous enhancement & $6(29 \%)$ \\
\hline & & Heterogeneous enhancement & $10(36 \%)$ \\
\hline & & $\begin{array}{c}\text { Central non enhancement with rim } \\
\text { enhancement }\end{array}$ & $10(36 \%)$ \\
\hline & $T a b$ & T and MRI Characteristics & \\
\hline
\end{tabular}

\begin{tabular}{|ccc|}
\hline Site of Involvement* & Number & Percentage (\%) \\
Face & 114 & 57 \\
Orbit & 196 & 93 \\
Orbital apex & 94 & 47 \\
Pterygopalatine fossa & 90 & 45 \\
Cavernous sinus & 9 & 4.6 \\
Internal carotid artery & 5 & 2.6 \\
Brain & 52 & 26 \\
Base & 200 & \\
\hline Table 3. Involvement of Extrasinus Structures (Stage 2 and 3 Diseases) \\
\hline
\end{tabular}




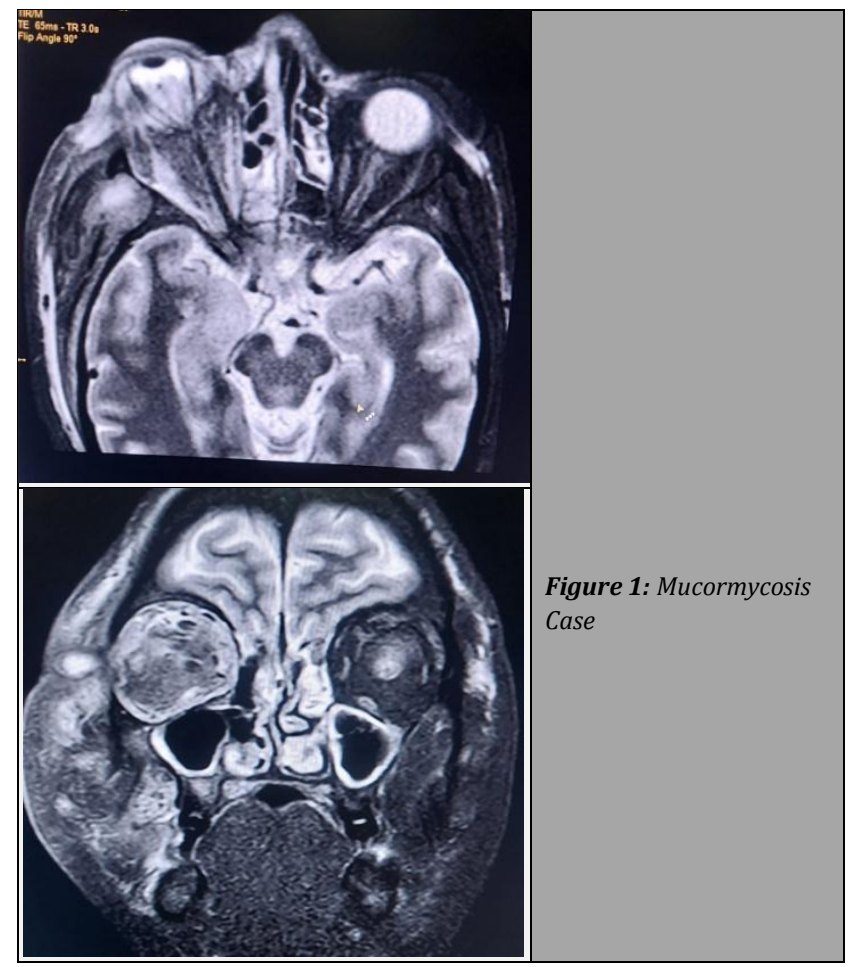

Figure 1 Axial and coronal fat suppressed STIR MR images of mucormycosis patient showing marked bilateral preseptal and periorbital soft tissue oedema and inflammation with diffuse thickening, oedema and inflammation in intraconal and extraconal compartments of bilateral orbit involving extraocular muscles with mild inflammation in the retro-orbital region with bilateral optic neuritis with right cavernous sinus thrombosis.

\section{DISCUSSION}

Several factors such as pre-existing diseases (DM, other respiratory diseases, immunosuppressant use, nosocomial infections and corticosteroid use with altered conditions of Covid can cause rhino-orbito-cerebral mucormycosis. ${ }^{5}$

Currently recommended intravenous methylprednisolone $0.5-1 \mathrm{mg} / \mathrm{kg} /$ day X 3days in moderate cases and $1-2$ $\mathrm{mg} / \mathrm{kg} /$ day in severe cases are used. The National Institute of Health advocates the use of dexamethasone $(6 \mathrm{mg}$ per day for a maximum of 10 days) in patients on a ventilator or with oxygen requirements. The risk of developing a secondary infection is seen in these cases too.

The factors of Covid-19 which cause mucormycosis are alveolar interstitial pathology and widespread lung involvement. Also, immune dysregulation seen with Covid-19 including decreased CD4 and CD8 cells alter immunity.

200 patients with suspected mucormycosis who underwent CT and MRI scanning were included to study the disease. In the majority of the cases, multiple sinuses were involved with isodense contents on CT scanning. Bony involvement and erosion were seen better on CT. On MRI, majority of the cases showed hyperintense contents on STIR images. Restriction on DWI MRI was seen in 74 patients. Extra sinus involvement particularly of the orbit was very common. The exact extent of the disease as well as extra sinus extension and intracerebral involvement was better seen on MRI.

An example of a mucormycosis case is given in figure 1.

\section{CONCLUSIONS}

Due to immune modulation secondary infections are seen in Covid-19 patients. In addition to this widespread use of corticosteroids and antibiotics increase propensity to this infection. Clinicians should have a high suspicion for mucormycosis in patients on corticosteroid therapy. We should try to achieve the required results through minimum dose and duration of corticosteroid therapy. Broad-spectrum antibiotic use should also be re-evaluated.

Imaging on MRI shows variable T2W signal intensity and sinus involvement. In our study, the patients had generally extensive disease. CT and MRI are important tools in early diagnosis of the disease and assessing the extent and complications. Proper treatment can be timely started and complications can be prevented with these.

Data sharing statement provided by the authors is available with the full text of this article at jemds.com.

Financial or other competing interests: None.

Disclosure forms provided by the authors are available with the full text of this article at jemds.com.

\section{REFERENCES}

[1] Casqueiro J, Casqueiro J, Alves C. Infections in patients with diabetes mellitus: a review of pathogenesis. Indian J Endocrinol Metab 2012;16 Suppl 1:S27-36.

[2] Sarrami AH, Setareh M, Izadinejad M, et al. Fatal disseminated mucormycosis in an immunocompetent patient: a case report and literature review. Int J Prev Med 2013;4(12):1468-71.

[3] Herrera DA, Dublin AB, Ormsby EL, et al. Imaging findings of rhinocerebralmucormycosis. Skull Base 2009;19(2):117-25.

[4] Vijayabala GS, Annigeri RG, Sudarshan R. Mucormycosis in a diabetic ketoacidosis patient. Asian Pac J Trop Biomed 2013;3(10):830-3.

[5] Atkins RC, Zimmet P. Diabetic kidney disease: act now or pay later. Saudi J Kidney Dis Transpl 2010;21(2):217-21. 